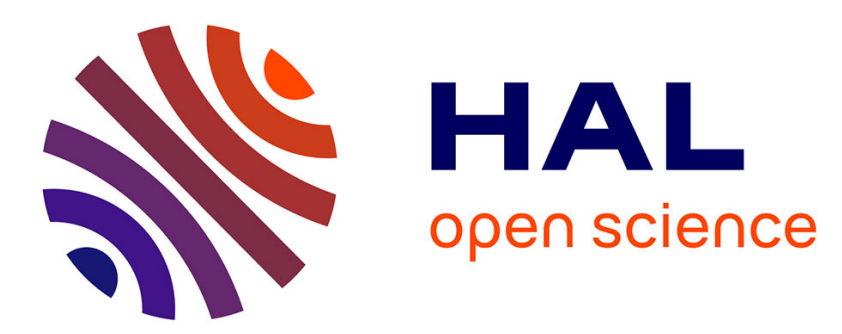

\title{
Bilinear control of high frequencies for a 1D Schrödinger equation
}

\author{
Karine Beauchard, Camille Laurent
}

\section{To cite this version:}

Karine Beauchard, Camille Laurent. Bilinear control of high frequencies for a 1D Schrödinger equation. Mathematics of Control, Signals, and Systems, 2017, 29 (2), pp.article 11. 10.1007/s00498-017-01878. hal-01333625

\section{HAL Id: hal-01333625 \\ https://hal.science/hal-01333625}

Submitted on 17 Jun 2016

HAL is a multi-disciplinary open access archive for the deposit and dissemination of scientific research documents, whether they are published or not. The documents may come from teaching and research institutions in France or abroad, or from public or private research centers.
L'archive ouverte pluridisciplinaire HAL, est destinée au dépôt et à la diffusion de documents scientifiques de niveau recherche, publiés ou non, émanant des établissements d'enseignement et de recherche français ou étrangers, des laboratoires publics ou privés. 


\title{
Bilinear control of high frequencies for a 1D Schrödinger equation
}

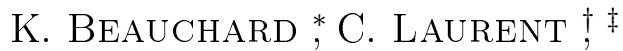

\begin{abstract}
In this article, we consider a $1 \mathrm{D}$ linear Schrödinger equation with potential $V$ and bilinear control. Under appropriate assumptions on $V$, we prove the exact controllability of high frequencies, in $H^{3}$, locally around any $H^{3}$-trajectory of the free system. In particular, any initial state in $H^{3}$ can be steered to a regular state, for instance a finite sum of eigenfunctions of $(-\Delta+V)$. This fact, coupled with a previous result due to Nersesyan, proves the global exact controllability of the system in $H^{3}$, with smooth controls, under appropriate assumptions.
\end{abstract}

\section{Introduction}

\subsection{Main result}

In this article, we consider the 1D Schrödinger equation

$$
\begin{cases}i \partial_{t} \psi(t, x)=\left(-\partial_{x}^{2}+V(x)-u(t) \mu(x)\right) \psi(t, x), & (t, x) \in(0, T) \times(0,1), \\ \psi(t, 0)=\psi(t, 1)=0, & t \in(0, T), \\ \psi(0, x)=\psi_{0}(x), & x \in(0,1),\end{cases}
$$

where $V, \mu \in L^{\infty}((0,1), \mathbb{R})$ and $u:(0, T) \rightarrow \mathbb{R}$. It is a bilinear control system in which the state is $\psi$ and the control is $u$.

To state our results, we first need to introduce few notations and recall well posedness results. We denote by $\langle.,$.$\rangle the L^{2}((0,1), \mathbb{C})$-scalar product,

$$
\langle f, g\rangle:=\Re\left(\int_{0}^{1} f(x) \overline{g(x)} d x\right),
$$

by $A_{V}$ the operator

$$
D\left(A_{V}\right):=H^{2} \cap H_{0}^{1}((0,1), \mathbb{C}), \quad A_{V}:=-\partial_{x}^{2}+V,
$$

${ }^{*}$ IRMAR and ENS Rennes, Avenue Robert Schumann, 35170 BRUZ, France, email: Karine.Beauchard@ens-rennes.fr

${ }^{\dagger}$ Laboratoire Jacques-Louis Lions, Université Pierre et Marie Curie, Boite courrier 38, 75252 Paris Cedex 05, France, email : laurent@ann.jussieu.fr

¥The authors were partially supported by the "Agence Nationale de la Recherche" (ANR), Projet Blanc EMAQS number ANR-2011-BS01-017-01. 
(which is assumed to be positive: replacing $V(x)$ by $V(x)+C$ which only affects the global phase of $\psi$ ) by $\left(\lambda_{k, V}\right)_{k \in \mathbb{N}^{*}}$ the nondecreasing sequence of its eigenvalues, by $\left(\varphi_{k, V}\right)_{k \in \mathbb{N}^{*}}$ associated eigenfunctions,

$$
\left\{\begin{array}{l}
-\varphi_{k, V}^{\prime \prime}(x)+V(x) \varphi_{k, V}(x)=\lambda_{k, V} \varphi_{k, V}(x), \quad x \in(0,1), \\
\varphi_{k, V}(0)=\varphi_{k, V}(1)=0 \\
\left\|\varphi_{k, V}\right\|_{L^{2}(0,1)}=1
\end{array}\right.
$$

by $\mathbb{P}_{K, V}$, for $K \in \mathbb{N}^{*}$, the projection

$$
\begin{array}{|ccc}
\mathbb{P}_{K, V}: \quad L^{2}((0,1), \mathbb{C}) & \rightarrow \overline{\operatorname{Span}_{\mathbb{C}}\left(\varphi_{k, V} ; k \geqslant K\right)}, \\
\xi & \mapsto \xi-\sum_{k=1}^{K-1}\left\langle\xi, \varphi_{k, V}\right\rangle \varphi_{k, V}
\end{array}
$$

by $H_{(V)}^{s}(0,1)$, for $s>0$, the Sobolev spaces

$$
H_{(V)}^{s}(0,1):=D\left(A_{V}^{s / 2}\right), \quad\|\xi\|_{H_{(V)}^{s}}:=\left(\sum_{k=1}^{\infty} \lambda_{k, V}^{s}\left|\left\langle\xi, \varphi_{k, V}\right\rangle\right|^{2}\right)^{1 / 2},
$$

which satisfy, in particular,

$$
H_{(V)}^{3}(0,1)=H_{(0)}^{3}(0,1)=\left\{\xi \in H^{3}((0,1), \mathbb{C}) ; \xi=\xi^{\prime \prime}=0 \text { at } x=0,1\right\},
$$

and by $\mathcal{S}$ the unitary $L^{2}((0,1), \mathbb{C})$-sphere. The following well-posedness result is a consequence of [3, Lemma 1] and the usual fixed point strategy (see [3, Proposition 3] for a the proof with $V=0$ ).

Proposition 1. Let $T>0, V, \mu \in H^{3}((0,1), \mathbb{R}), \psi_{0} \in H_{(0)}^{3}((0,1), \mathbb{C})$ and $u \in L^{2}((0, T), \mathbb{R})$. There exists a unique solution $\psi \in C^{0}\left([0, T], H_{(0)}^{3}(0,1)\right)$ of (1). Moreover $\|\psi(t)\|_{L^{2}(0,1)}=\left\|\psi_{0}\right\|_{L^{2}}$ for every $t \in[0, T]$.

The goal of this article is the proof of the following result.

Theorem 1. Let $T>0, \psi_{0} \in H_{(0)}^{3}((0,1), \mathbb{C}) \cap \mathcal{S}, \psi_{\text {ref }}(t):=e^{-i A_{V} t} \psi_{0}$, and $V, \mu \in H^{3}((0,1), \mathbb{R})$ be such that

$$
A_{V} \text { has a simple spectrum, }
$$

$$
\begin{gathered}
\mu^{\prime}(1) \varphi_{k, V}^{\prime}(1)+\mu^{\prime}(0) \varphi_{k, V}^{\prime}(0) \neq 0 \text { and } \\
\mu^{\prime}(1) \varphi_{k, V}^{\prime}(1)-\mu^{\prime}(0) \varphi_{k, V}^{\prime}(0) \neq 0, \text { for every } k \in \mathbb{N}^{*} .
\end{gathered}
$$

1. There exists $K \in \mathbb{N}^{*}, \delta>0$ and a $C^{1}$-map

$$
\Gamma: \mathcal{V} \rightarrow L^{2}((0, T), \mathbb{R})
$$

where

$$
\mathcal{V}:=\left\{\psi_{f} \in \mathbb{P}_{K, V}\left[H_{(0)}^{3}(0,1)\right] ;\left\|\psi_{f}-\mathbb{P}_{K, V}\left[\psi_{r e f}(T)\right]\right\|_{H_{(0)}^{3}}<\delta\right\}
$$

such that

- $\Gamma\left(\mathbb{P}_{K, V}\left[\psi_{r e f}(T)\right]\right)=0$, 
- for every $\psi_{f} \in \mathcal{V}$ the solution of (1) with control $u=\Gamma\left[\psi_{f}\right]$ satisfies $\mathbb{P}_{K, V}[\psi(T)]=\psi_{f}$.

2. As a consequence, there exists $K^{\prime} \geqslant K$ and $u \in L^{2}((0, T), \mathbb{R})$ such that the solution of (1) satisfies $\mathcal{P}_{K^{\prime}, V}[\psi(T)]=0$; in particular, $\psi(T,.) \in$ $H_{(V)}^{4}((0,1), \mathbb{C})$.

This result allows to prove the global exact controllability of $\sqrt{1})$ in $H_{(V)}^{3}((0,1), \mathbb{C})$, instead of $H_{(V)}^{4}((0,1), \mathbb{C})$ in [13] $\left(\right.$ or $H_{(V)}^{3+}((0,1), \mathbb{C})$ as can be proved by following the original proof [15]).

Corollary 1. Let $V, \mu \in H^{4}((0,1), \mathbb{R})$ that satisfy (5) and

$$
\begin{gathered}
\exists C>0 \text { such that }\left|\left\langle\mu \varphi_{1, V}, \varphi_{k, V}\right\rangle\right| \geqslant \frac{C}{k^{3}}, \forall k \in \mathbb{N}^{*}, \\
\lambda_{k, V}-\lambda_{1, V} \neq \lambda_{p, V}-\lambda_{q, V}, \forall k, p, q \in \mathbb{N}^{*} \text { such that }\{1, k\} \neq\{p, q\} .
\end{gathered}
$$

For every $\psi_{0}, \psi_{f} \in H_{(V)}^{3}((0,1), \mathbb{C}) \cap \mathcal{S}$, there exists $T>0$ and $u \in L^{2}((0, T), \mathbb{R})$ such that the solution of (1) satisfies $\psi(T)=\psi_{f}$.

\subsection{Bibliographical comments}

The Schrödinger equation with bilinear control has been widely studied in the litterature. The multi-d model writes

$$
\begin{cases}\left(i \partial_{t}+\Delta-V\right) \psi(t, x)=u(t) \mu(x) \psi(t, x), & (t, x) \in(0, T) \times \Omega, \\ \psi(t, x)=0, & (t, x) \in(0, T) \times \partial \Omega,\end{cases}
$$

where $\Omega$ is a bounded open subset of $\mathbb{R}^{N}, N \in \mathbb{N}^{*}, V, \mu: \Omega \rightarrow \mathbb{R}$ are given functions, the state $\psi$ lives in the $L^{2}(\Omega, \mathbb{C})$-sphere, denoted $\mathcal{S}$ and the control is the real valued function $u:(0, T) \rightarrow \mathbb{R}$.

A negative result A negative control result was proved by Turinici in [18, as a consequence of a general result by Ball, Marsden and Slemrod in [1]. It states that, for $V=0$, for a given function $\mu \in C^{2}(\Omega, \mathbb{R})$, for a given initial condition $\psi_{0} \in\left(H^{2} \cap H_{0}^{1}\right)(\Omega, \mathbb{C}) \cap \mathcal{S}$, and by using controls $u \in L_{l o c}^{r}((0, \infty), \mathbb{R})$ with $r>1$, one may only reach a subset of $\left(H^{2} \cap H_{0}^{1}\right)(\Omega) \cap \mathcal{S}$ that has an empty interior in $\left(H^{2} \cap H_{0}^{1}\right)(\Omega, \mathbb{C}) \cap \mathcal{S}$. Recently, Boussaid, Caponigro and Chambrion extended this negative result to the case of controls in $L_{l o c}^{1}((0, \infty), \mathbb{R})$, see [7]. However, this negative result is actually due to a bad choice of functional setting, as emphasized in the next paragraph.

Local exact results in 1-d Beauchard proved in 2] the exact controllability of equation (8), locally around the ground state in $H^{7}$, with controls $u \in H^{1}((0, T), \mathbb{R})$ in large time $T$, in the case $N=1, \Omega=(-1 / 2,1 / 2), \mu(x)=x$ and $V=0$. The proof of 2 relies on Coron's return method and Nash-Moser theorem.

Reference [3] improves this result and establishes the exact controllability of equation (8), locally around the ground state in $H^{3}$, with controls $u \in$ $L^{2}((0, T), \mathbb{R})$, in arbitrary time $T>0$, and with generic functions $\mu$ when $N=1$, $\Omega=(0,1), V=0$. This result can be extended to an arbitrary potential $V$, as 
explained in [13]. The proof relies on a smoothing effect, that allows to conclude with the inverse mapping theorem (instead of Nash-Moser's one).

Then, Morancey and Nersesyan developped this stategy to control a Schrödinger equation with a polarizability term [12] and a finite number of Schrödinger equations with one control [11, 13].

Global approximate results in $\mathbf{N}-\mathbf{d}$ Three strategies have been developed to study approximate controllability for equation (8)

The first strategy is a variationnal argument introduced by Nesesyan in 14. It proves, under appropriate assumptions on $(V, \mu)$, that any initial condition in $H_{(0)}^{3+}(\Omega, \mathbb{C}) \cap \mathcal{S}$ can be steered to the ground state, approximately in $H^{3}$, with smooth controls $u \in C_{c}^{\infty}((0, T), \mathbb{R})$, in large time $T$, in arbitrary dimension $N$.

Note that, in 1D, this result can be coupled with the previous local exact controllability results. Then, under appropriate assumptions on $(V, \mu)$, we get that any initial condition in $H_{(0)}^{3+}((0,1), \mathbb{C}) \cap \mathcal{S}$ can be steered to the ground state, exactly, in large time $T>0$, with controls $u \in L^{2}((0, T), \mathbb{R})$. See $[15]$ for one equation (8), [12] for a Schrödinger equation with a polarizability term, [13] for a finite number of Schrödinger equations with the same control.

A second strategy consists in deducing approximate controllability in regular spaces (containing $H^{3}$ ) from exact controllability results in infinite time by Nersesyan and Nersisyan [9]

A third strategy, due to Chambrion, Mason, Sigalotti, and Boscain [8, relies on geometric techniques for the controllability of the Galerkin approximations. It proves (under appropriate assumptions on $V$ and $\mu$ ) the approximate controllability of $(8)$ in $L^{2}$, with piece-wise constant controls. The hypotheses of this result were refined by Boscain, Caponigro, Chambrion, and Sigalotti in [4. The approximate controllability is proved in higher Sobolev norms in 7 for one equation, and in [6] for a finite number of equations with one control. For more details and more references about the geometric techniques, we refer the reader to the recent survey [5].

\subsection{Structure of this article}

In Section 2, we give the main steps of the proof of Theorem 1. Two intermediary results are stated and used in this proof, but proved later, in Sections 3 and 4 . Finally, in Section 5 , we prove Corollary 1 .

\section{Proof of the main result}

In this section $V, \mu, T, \psi_{0}, \psi_{\text {ref }}$ are fixed and satisfy the assumptions of Theorem 1. The first statement of this theorem comes by applying the inverse mapping theorem to the map

$$
\begin{aligned}
\Theta_{K}: \quad L^{2}((0, T), \mathbb{R}) & \rightarrow \mathbb{P}_{K, V}\left[H_{(0)}^{3}(0,1)\right] \\
u & \mapsto \mathbb{P}_{K, V}[\psi(T)]
\end{aligned}
$$

where $\psi$ solves (1). Adapting the proof of [3, Proposition 3] to the case $V \neq 0$, we see that $\Theta_{K}$ is a $C^{1}$-map and

$$
\begin{aligned}
d \Theta_{K}(0): \quad L^{2}((0, T), \mathbb{R}) & \rightarrow \mathbb{P}_{K, V}\left[H_{(0)}^{3}(0,1)\right] \\
v & \mapsto \mathbb{P}_{K, V}[\Psi(T)]
\end{aligned}
$$


where $\Psi$ solves the linearized system

$$
\begin{cases}i \partial_{t} \Psi(t, x)=\left(-\partial_{x}^{2}+V(x)\right) \Psi(t, x)-v(t) \mu(x) \psi_{\text {ref }}(t, x), & (t, x) \in(0, T) \times(0,1), \\ \Psi(t, 0)=\Psi(t, 1), & t \in(0, T), \\ \Psi(0, x)=0, & x \in(0,1) .\end{cases}
$$

Thus, to prove Theorem 11 1 , it suffices to prove that, for $K$ large enough, $d \Theta_{K}(0)$ has a continuous right-inverse between the following spaces

$$
d \Theta_{K}(0)^{-1}: \mathbb{P}_{K, V}\left[H_{(0)}^{3}(0,1)\right] \rightarrow L^{2}((0, T), \mathbb{R}) .
$$

To this aim, we introduce the decomposition $\mu(x) \psi_{\text {ref }}(t, x)=\left(\mu_{1}+\mu_{2}\right)(t, x)$ where $\mu_{2} \in C^{0}\left([0, T], H_{(0)}^{3}(0,1)\right)$ solves

$$
\begin{cases}\left(-\partial_{x}^{2}+V\right)^{2} \mu_{2}=\left(-\partial_{x}^{2}+V\right)^{2}\left[\mu \psi_{r e f}\right], & (t, x) \in(0, T) \times(0,1), \\ \mu_{2}(t, \sigma)=\partial_{x}^{2} \mu_{2}(t, \sigma)=0, & (t, \sigma) \in(0, T) \times\{0,1\},\end{cases}
$$

and

$$
\mu_{1}(t, x):=\mu(x) \psi_{r e f}(t, x)-\mu_{2}(t, x), \quad \forall(t, x) \in(0, T) \times(0,1),
$$

i.e.

$$
\begin{cases}\left(-\partial_{x}^{2}+V\right)^{2} \mu_{1}=0, & (t, x) \in(0, T) \times(0,1) \\ \mu_{1}(t, \sigma)=0, & (t, \sigma) \in(0, T) \times\{0,1\} \\ \partial_{x}^{2} \mu_{1}(t, \sigma)=2 \mu^{\prime}(\sigma) \partial_{x} \psi_{r e f}(t, \sigma) & (t, \sigma) \in(0, T) \times\{0,1\} .\end{cases}
$$

This decomposition is inspired by [17. Then,

$$
d \Theta_{K}(0) \cdot v=\left(\mathcal{L}_{K}+\mathcal{K}_{K}\right)(v)
$$

where

$$
\begin{aligned}
\mathcal{L}_{K}: \quad L^{2}((0, T), \mathbb{R}) & \rightarrow \mathbb{P}_{K, V}\left[H_{(0)}^{3}(0,1)\right] \\
v & \mapsto \mathbb{P}_{K, V}\left[\Psi_{1}(T)\right] \\
\mathcal{K}_{K}: \quad L^{2}((0, T), \mathbb{R}) & \rightarrow \mathbb{P}_{K, V}\left[H_{(0)}^{3}(0,1)\right] \\
v & \mapsto \mathbb{P}_{K, V}\left[\Psi_{2}(T)\right]
\end{aligned}
$$

and, for $j=1,2$,

$$
\begin{cases}i \partial_{t} \Psi_{j}(t, x)=\left(-\partial_{x}^{2}+V(x)\right) \Psi_{j}(t, x)-v(t) \mu_{j}(t, x), & (t, x) \in(0, T) \times(0,1), \\ \Psi_{j}(t, 0)=\Psi_{j}(t, 1), & t \in(0, T), \\ \Psi_{j}(0, x)=0, & x \in(0,1) .\end{cases}
$$

By [3. Lemma 1], for every $v \in L^{2}((0, T), \mathbb{R}), \Psi_{j} \in C^{0}\left([0, T], H_{(0)}^{3}(0,1)\right)$ and thus $\mathcal{L}_{K}, \mathcal{K}_{K}$ are continuous operators. The following 2 results will be proved in Sections 3 and 4

Proposition 2. There exists $K_{*} \in \mathbb{N}^{*}, \mathcal{C}>0$ and a decreasing sequence $\left(\mathcal{H}_{K}\right)_{K \geqslant K_{*}}$ of closed vector subspaces of $L^{2}((0, T), \mathbb{R})$ satisfying

$$
\underset{K \geqslant K^{*}}{\cap} \mathcal{H}_{K}=\{0\},
$$

such that for every $K \geqslant K_{*}$, the operator $\mathcal{L}_{K}: \mathcal{H}_{K} \rightarrow \mathbb{P}_{K}\left[H_{(0)}^{3}(0,1)\right]$ is an isomorphism and

$$
\left\|\mathcal{L}_{K}^{-1}\right\|_{\mathbb{P}_{K, V}\left[H_{(0)}^{3}\right] \rightarrow L^{2}} \leqslant \mathcal{C} .
$$


Proposition 3. For every $K \in \mathbb{N}^{*}$, the operator $\mathcal{K}_{K}: L^{2}((0, T), \mathbb{R}) \rightarrow \mathbb{P}_{K}\left[H_{(0)}^{3}(0,1)\right]$ is compact.

To end the proof of Theorem 1.1, it suffices to prove the existence of $K \geqslant K_{*}$ such that $d \Theta_{K}(0)=\left(\mathcal{L}_{K}+\mathcal{K}_{K}\right)$ is an isomorphism from $\mathcal{H}_{K}$ to $\mathbb{P}_{K, V}\left[H_{(0)}^{3}(0,1)\right]$.

Working by contradiction, we assume that, for every $K \geqslant K_{*},\left(\mathcal{L}_{K}+\mathcal{K}_{K}\right)$ : $\mathcal{H}_{K} \rightarrow \mathbb{P}_{K}\left[H_{(0)}^{3}(0,1)\right]$ is not an isomorphism. By Fredholm aternative, there exists a sequence $\left(v_{K}\right)_{K \geqslant K_{*}}$ such that

$$
v_{K} \in \mathcal{H}_{K}, \quad\left\|v_{K}\right\|_{L^{2}}=1, \quad\left(\mathcal{L}_{K}+\mathcal{K}_{K}\right)\left(v_{K}\right)=0, \quad \forall K \geqslant K_{*} .
$$

Then, by 14

$$
v_{K} \rightarrow 0 \text { in } L^{2}((0, T), \mathbb{R})
$$

and

$$
\begin{aligned}
1 & =\left\|v_{K}\right\|_{L^{2}(0, T)}=\left\|\mathcal{L}_{K}^{-1} \circ \mathcal{L}_{K}\left(v_{K}\right)\right\|_{L^{2}(0, T)} \text { because } v_{K} \in \mathcal{H}_{K} \\
& \leqslant \mathcal{C}\left\|\mathcal{L}_{K}\left(v_{K}\right)\right\|_{H_{(0)}^{3}(0,1)} \text { by } 15 \\
& \leqslant \mathcal{C}\left\|\mathcal{K}_{K}\left(v_{K}\right)\right\|_{H_{(0)}^{3}(0,1)} \\
& \leqslant \mathcal{C}\left\|\mathcal{K}_{1}\left(v_{K}\right)\right\|_{H_{(0)}^{3}(0,1)}^{\longrightarrow} 0
\end{aligned}
$$

because $\mathcal{K}_{1}$ is compact. This is a contradiction.

To prove the second statement of Theorem 1 1 one considers $K^{\prime} \geqslant K$ such that $\left\|\mathbb{P}_{K^{\prime}, V}\left(\psi_{\text {ref }}(T)\right)\right\|_{H_{(0)}^{3}}<\delta$ and applies statement 1 to $\psi_{f}:=\left(\mathbb{P}_{K, V}-\right.$ $\left.\mathbb{P}_{K^{\prime}, V}\right)\left[\psi_{\text {ref }}(T)\right]$.

\section{Ingham inequality}

The goal of this section is to prove Proposition 2 by reducing the problem to an Ingham inequality. First, we recall useful estimates (see [16, Theorem 4]).

$$
\begin{gathered}
\lambda_{k, V}=(k \pi)^{2}+\int_{0}^{1} V(x) d x+r_{k} \quad \text { where } \sum_{k=1}^{\infty} r_{k}^{2}<\infty, \\
\exists C=C(V)>0 \text { such that }\left\|\varphi_{k, V}^{\prime}-\varphi_{k, 0}^{\prime}\right\|_{L^{\infty}(0,1)} \leqslant C, \quad \forall k \in \mathbb{N}^{*} .
\end{gathered}
$$

By the Duhamel formula, we have

$$
\Psi_{j}(T)=i \sum_{k=1}^{\infty} \int_{0}^{T} v(t)\left\langle\mu_{j}(t), \varphi_{k, V}\right\rangle e^{-i \lambda_{k, V}(T-t)} d t \varphi_{k, V} .
$$

For every $t \in(0, T)$, the function $x \mapsto \mu_{1}(t, x)$ solves a ordinary differential equation of order 4 with continuous coefficients, because $V \in H^{3}((0,1), \mathbb{R})$ (see $(12)$ ), thus $\mu_{1}(t,.) \in C^{4}([0,1], \mathbb{C})$ and the following integrations by parts are legitimate

$$
\begin{aligned}
\left\langle\mu_{1}(t), \varphi_{k}\right\rangle & =\frac{1}{\lambda_{k, V}^{2}} \int_{0}^{1} \mu_{1}(t, x)\left(-\partial_{x}^{2}+V(x)\right)^{2} \varphi_{k, V}(x) d x \\
& =\frac{2}{\lambda_{k, V}^{2}}\left(\mu^{\prime}(1) \partial_{x} \psi_{r e f}(t, 1) \varphi_{k, V}^{\prime}(1)-\mu^{\prime}(0) \partial_{x} \psi_{r e f}(t, 0) \varphi_{k, V}^{\prime}(0)\right) .
\end{aligned}
$$


Thus, for a given target $\Psi_{f} \in \mathbb{P}_{K}\left[H_{(0)}^{3}(0,1)\right]$ and a fonction $v \in L^{2}((0, T), \mathbb{R})$, the equality $\mathcal{L}_{K}(v)=\Psi_{f}$ is equivalent to the moment problem

$$
\int_{0}^{T} v(t) f_{k, V}(t) d t=\frac{\lambda_{k, V}^{2}}{2 k \pi}\left\langle\Psi_{f}, \varphi_{k, V}\right\rangle e^{i \lambda_{k, V} T}, \quad \forall k \geqslant K,
$$

where

$f_{k, V}(t):=\left(\mu^{\prime}(1) \partial_{x} \psi_{r e f}(t, 1) \varphi_{k, V}^{\prime}(1)-\mu^{\prime}(0) \partial_{x} \psi_{r e f}(t, 0) \varphi_{k, V}^{\prime}(0)\right) \frac{e^{i \lambda_{k, V} t}}{k \pi}, \forall k \in \mathbb{N}^{*}$.

Note that the right hand side of 200 belongs to $l^{2}$ thanks to 17$)$ and (3). Let

$$
\mathcal{H}_{K}^{\mathbb{C}}:=\operatorname{Adh}_{L^{2}((0, T), \mathbb{C})}\left(\operatorname{Vect}\left\{f_{k} ;|k| \geqslant K\right) \text { and } \mathcal{H}_{K}:=\mathcal{H}_{K}^{\mathbb{C}} \cap L^{2}((0, T), \mathbb{R})\right.
$$

where $f_{k}(t):=\overline{f_{-k}(t)}, \forall k \leqslant-1$. Clearly, $(14)$ is satisfied. The following Ingham inequality - that will be proved later on - proves that, for $K$ large enough, $\left(f_{k}\right)_{|k| \geqslant K}$ is a Riesz basis of $\mathcal{H}_{K}^{\mathbb{C}}$.

Proposition 4. There exists $K_{*} \in \mathbb{N}^{*}$ and $\mathcal{C}_{1}, \mathcal{C}_{2}>0$ such that

$$
\mathcal{C}_{1}\|b\|_{l^{2}} \leqslant\left(\int_{0}^{T}\left|\sum_{|k| \geqslant K_{*}} b_{k} f_{k}(t)\right|^{2} d t\right)^{1 / 2} \leqslant \mathcal{C}_{2}\|b\|_{l^{2}}, \quad \forall b \in l^{2}\left(\mathbb{Z}_{K_{*}}, \mathbb{C}\right),
$$

where $\mathbb{Z}_{K_{*}}:=\left\{k \in \mathbb{Z} ;|k| \geqslant K_{*}\right\}$.

This proposition has 3 consequences: for every $K \geqslant K_{*}$

- for every $\left(d_{k}\right)_{|k| \geqslant K} \in l^{2}\left(\mathbb{Z}_{K}, \mathbb{C}\right)$, there exists a unique function $v \in \mathcal{H}_{K}^{\mathbb{C}}$ such that

$$
\int_{0}^{T} v(t) f_{k}(t) d t=d_{k}, \quad \forall|k| \geqslant K
$$

- in particular, if $d_{-k}=\overline{d_{k}}$ for every $k$ then $v$ is real valued (consequence of uniqueness); this proves that $\mathcal{L}_{K}: \mathcal{H}_{K} \rightarrow \mathbb{P}_{K, V}\left[H_{(0)}^{3}(0,1)\right]$ is bijective,

- moreover, this candidate is the unique solution in $L^{2}((0, T), \mathbb{R})$ of the moment problem 23 with minimal $L^{2}(0, T)$-norm; this proves that the sequence $\left(\left\|\mathcal{L}_{K}^{-1}\right\|_{\mathbb{P}_{K, V}\left[H_{(0)}^{3}\right] \rightarrow \mathcal{H}_{K}}\right)_{K \geqslant K_{*}}$ is decreasing and thus 15 holds.

which ends the proof of Proposition 2.

Proof of Proposition 4

Step 1: We prove that the 2 functions $g_{ \pm}:(0, T) \rightarrow \mathbb{C}$ defined by

$$
g_{ \pm}(t):=\mu^{\prime}(1) \partial_{x} \psi_{r e f}(t, 1) \pm \mu^{\prime}(0) \partial_{x} \psi_{r e f}(t, 0)
$$

do not vanish on $(0, T)$. It is a consequence of (4), (5) and the explicit expression

$$
g_{ \pm}(t)=\sum_{k=1}^{\infty}\left(\mu^{\prime}(1) \varphi_{k, V}^{\prime}(1) \pm \mu^{\prime}(0) \varphi_{k, V}^{\prime}(0)\right) e^{i \lambda_{k, V} t}
$$


Step 2: We prove the existence of $K_{0}, \mathcal{C}_{1}^{0}, \mathcal{C}_{2}^{0}>0$ such that

$$
\mathcal{C}_{1}^{0}\|b\|_{l^{2}} \leqslant\left(\int_{0}^{T}\left|\sum_{|k| \geqslant K_{0}} b_{k} h_{k}(t)\right|^{2} d t\right)^{1 / 2} \leqslant \mathcal{C}_{2}^{0}\|b\|_{l^{2}}, \quad \forall b \in l^{2}\left(\mathbb{Z}_{K_{0}}, \mathbb{C}\right),
$$

where

$$
h_{k}(t):=\left((-1)^{k} \mu^{\prime}(1) \partial_{x} \psi_{r e f}(t, 1)-\mu^{\prime}(0) \partial_{x} \psi_{r e f}(t, 0)\right) e^{i \lambda_{k}, v t}, \quad \forall k \in \mathbb{N}^{*},
$$

Thanks to (4) and (17), for every $0 \leqslant \tau_{1}<\tau_{2}<\infty$, there exists $\mathcal{C}_{j}^{\prime}=\mathcal{C}_{j}^{\prime}\left(\tau_{1}, \tau_{2}\right)>$ 0 such that

$$
\mathcal{C}_{1}^{\prime}\|b\|_{l^{2}} \leqslant\left(\int_{\tau_{1}}^{\tau_{2}}\left|\sum_{|k| \geqslant 1} b_{k} e^{ \pm i \lambda_{k, V} t}\right|^{2} d t\right)^{1 / 2} \leqslant \mathcal{C}_{2}^{\prime}\|b\|_{l^{2}}, \quad \forall b \in l^{2}(\mathbb{Z}-\{0\}, \mathbb{C}),
$$

where $\lambda_{k, V}:=-\lambda_{k, V}, \forall k \leqslant-1$ (see [10]). have

Let $\left(b_{k}\right)_{|k| \geqslant K}$ be a sequence of complex numbers with finite support. We

$$
\begin{aligned}
\left\|\sum_{|k| \geqslant K} b_{k} h_{k}\right\|_{L^{2}(0, T)}^{2} & =\left\|g_{+}(t) \sum_{|k| \text { odd } \geqslant K} b_{k} e^{i \lambda_{k, V} t}\right\|_{L^{2}(0, T)}^{2} \\
& +\left\|g_{-}(t) \sum_{|j| \text { even } \geqslant K} b_{j} e^{i \lambda_{j, V} t}\right\|_{L^{2}(0, T)}^{2} \\
& -2 \Re\left(\mathcal{T}_{K}\right)
\end{aligned}
$$

where

$$
\mathcal{T}_{K}:=\sum_{|k| \text { odd } \geqslant K} \sum_{|j| \text { even } \geqslant K} b_{k} \overline{b_{j}} \int_{0}^{T} g_{+}(t) g_{-}(t) e^{i\left(\lambda_{k, V}-\lambda_{j, V}\right) t} d t .
$$

For any $x \in[0,1]$, the map $t \mapsto \partial_{x} \psi_{r e f}(t, x)$ belongs to $H^{1}(0, T)$; indeed,

$$
\partial_{t} \partial_{x} \psi_{r e f}(t, x)=-i \sum_{k=1}^{\infty} \lambda_{k, V}\left\langle\psi_{0}, \varphi_{k, V}\right\rangle e^{-i \lambda_{k, V} t} \varphi_{k, V}^{\prime}(x)
$$

thus, by 18 and 25

$\int_{0}^{T}\left|\partial_{t} \partial_{x} \psi_{r e f}(t, x)\right|^{2} d t \leqslant \mathcal{C}_{2}^{\prime}(0, T)^{2} \sum_{k=1}^{\infty}\left|\lambda_{k, V}(k \pi+C)\left\langle\psi_{0}, \varphi_{k, V}\right\rangle\right|^{2} \leqslant C^{\prime}\left\|\psi_{0}\right\|_{H_{(0)}^{3}}^{2}$.

Therefore, the maps $g_{ \pm}$belong to $H^{1}((0, T), \mathbb{C})$, which is an algebra, thus there exists $C>0$ such that (integration by part)

$$
\left|\int_{0}^{T} g_{+}(t) g_{-}(t) e^{i \omega t} d t\right| \leqslant \frac{C}{|\omega|}, \quad \forall|\omega| \geqslant 1 .
$$

Then, by Cauchy-Schwarz inequality,

$$
\left|\mathcal{T}_{K}\right| \leqslant C\left(\sum_{|k| \text { odd } \geqslant K}\left|b_{k}\right|^{2}\right)^{1 / 2}\left(\sum_{|j| \text { even } \geqslant K}\left|b_{j}\right|^{2}\right)^{1 / 2} \sqrt{\epsilon_{K}}
$$


where

$$
\epsilon_{K}:=\sum_{|k| \text { odd } \geqslant K} \sum_{|j| \text { even } \geqslant K} \frac{1}{\left(\lambda_{k, V}-\lambda_{j, V}\right)^{2}} .
$$

is finite and converges to zero when $K \rightarrow \infty$. Indeed, by (17), there exists $C, K^{\prime}>0$ such that $\left|\lambda_{k, V}-\lambda_{j, V}\right| \geqslant C\left|k^{2}-j^{2}\right|$ for every odd integer $k \geqslant K^{\prime}$ and even integer $j \geqslant K^{\prime}$. Moreover, using the decomposition

$$
\frac{1}{\left(k^{2}-j^{2}\right)^{2}}=\frac{1}{4 k^{2}}\left(\frac{1}{(j-k)^{2}}+\frac{1}{(j+k)^{2}}\right)-\frac{1}{4 k^{3}}\left(\frac{1}{j-k}-\frac{1}{j+k}\right)
$$

we get

$$
\sum_{k \text { odd } \geqslant K} \sum_{\text {jeven } \geqslant K} \frac{1}{\left(k^{2}-j^{2}\right)^{2}} \leqslant C^{\prime} \sum_{k \text { odd } \geqslant K} \frac{1}{k^{2}} \leqslant \frac{C^{\prime \prime}}{K} .
$$

By Step 1, there exists $0 \leqslant \tau_{1}^{ \pm}<\tau_{2}^{ \pm} \leqslant T$ and $m>0$ such that $\left|g_{ \pm}(t)\right| \geqslant m$ for every $t \in\left(\tau_{1}^{ \pm}, \tau_{2}^{ \pm}\right)$. We deduce from $(26)$ and 25$)$ that

$$
\left\|\sum_{|k| \geqslant K} b_{k} h_{k}\right\|_{L^{2}(0, T)}^{2} \geqslant\left(A-C \sqrt{\epsilon_{K}}\right) \sum_{|k| \geqslant K}\left|b_{k}\right|^{2}
$$

where $A:=m^{2} \min \left\{\mathcal{C}_{1}^{\prime}\left(\tau_{1}^{+}, \tau_{2}^{+}\right)^{2} ; \mathcal{C}_{1}^{\prime}\left(\tau_{1}^{-}, \tau_{2}^{-}\right)^{2}\right\}$. This gives the lower bound of 24) with $K_{0}$ large enough so that $\mathcal{C}_{1}^{0}:=\sqrt{A-C \sqrt{\epsilon_{K_{0}}}}>0$. Let $M>0$ be such that $g_{ \pm}(t) \leqslant M$ for every $t \in(0, T)$. We deduce from $(26)$ and $(25)$ that the upper bound of 24 holds with $\mathcal{C}_{2}^{0}:=\sqrt{M \mathcal{C}_{2}^{\prime}(0, T)+C \sqrt{\epsilon_{K_{0}}}}$.

Step 3: Conclusion. By 18, there exists $C>0$ such that

$$
\left\|\sum_{|k| \geqslant K} b_{k}\left(f_{k}-h_{k}\right)\right\|_{L^{2}(0, T)} \leqslant C \sum_{|k| \geqslant K} \frac{\left|b_{k}\right|}{k} \leqslant C\left(\sum_{|k| \geqslant K}\left|b_{k}\right|^{2}\right)\left(\sum_{|k| \geqslant K} \frac{1}{k^{2}}\right) .
$$

We deduce from 24 that, for every $K \geqslant K_{0}$,

$$
\left(\mathcal{C}_{1}^{0}-\frac{2}{K-1}\right)\|b\|_{l^{2}} \leqslant\left(\int_{0}^{T}\left|\sum_{|k| \geqslant K_{\sharp}} b_{k} f_{k}\right|^{2} d t\right)^{1 / 2} \leqslant\left(\mathcal{C}_{2}^{0}+\frac{2}{K-1}\right)\|b\|_{l^{2}}
$$

which gives the conclusion with any $K_{*} \geqslant K_{0}$ large enough so that $\mathcal{C}_{1}$ := $\mathcal{C}_{1}^{0}-\frac{2}{K-1}>0$.

\section{Compactness property}

The goal of this section is to prove Proposition 3. Let $K \in \mathbb{N}^{*}$ and $\left(v_{n}\right)_{n \in \mathbb{N}}$ be a sequence in $L^{2}((0, T), \mathbb{R})$ that weakly converges to 0 , and is bounded by 1 . Then,

$$
\left\|\mathcal{K}_{K}\left(v_{n}\right)\right\|_{H_{(0)}^{3}}^{2}=\sum_{k \geqslant K}\left|(k \pi)^{3} \int_{0}^{T} v_{n}(t)\left\langle\mu_{2}(t), \varphi_{k, V}\right\rangle e^{i \lambda_{k, V} t} d t\right|^{2} .
$$


Each term of this sum converges to zero when $[n \rightarrow \infty]$. Moreover, using the explicit expression $\varphi_{k, 0}(x)=\sqrt{2} \sin (k \pi x)$, integrations by part (note that $\left.\mu_{2} \in C^{0}\left([0, T], H_{(0)}^{3}(0,1)\right)\right)$, Cauchy-Schwarz inequality and $(18)$, we get

$$
\begin{aligned}
& \left|(k \pi)^{3} \int_{0}^{T} v_{n}(t)\left\langle\mu_{2}(t), \varphi_{k, V}\right\rangle e^{i \lambda_{k, V} t} d t\right| \\
\leqslant & C\left|k^{3} \int_{0}^{T} v_{n}(t)\left\langle\mu_{2}(t), \varphi_{k, 0}\right\rangle e^{i \lambda_{k, V} t} d t\right|+C\left|k^{3} \int_{0}^{T} v_{n}(t)\left\langle\mu_{2}(t), \varphi_{k, V}-\varphi_{k, 0}\right\rangle e^{i \lambda_{k, V} t} d t\right| \\
\leqslant & C\left|\int_{0}^{T} v_{n}(t)\left\langle\partial_{x}^{3} \mu_{2}(t), \sqrt{2} \cos (k \pi x)\right\rangle e^{i \lambda_{k, V} t} d t\right|+\frac{C}{k} \int_{0}^{T}\left|v_{n}(t)\right|\left\|\mu_{2}(t)\right\|_{H_{(0)}^{3}} d t \\
\leqslant & C\left(\int_{0}^{T}\left|\left\langle\partial_{x}^{3} \mu_{2}(t), \sqrt{2} \cos (k \pi x)\right\rangle\right|^{2} d t\right)^{1 / 2}+\frac{C}{k}\left(\int_{0}^{T}\left\|\mu_{2}(t)\right\|_{H_{(0)}^{3}}^{2} d t\right)^{1 / 2} .
\end{aligned}
$$

This right-hand side belongs to $l^{2}\left(\mathbb{Z}_{K}\right)$ and does not depend on $n$, thus, by the dominated convergence theorem $\mathcal{K}_{K}\left(v_{n}\right) \underset{n \rightarrow \infty}{\longrightarrow} 0$ in $H_{(0)}^{3}(0,1)$.

\section{Global exact controllability in $H_{(0)}^{3}(0,1)$}

The following result is proved in 13 , Theorem 5.1], by following the proof developped in the original article [15].

Proposition 5. Let $V, \mu \in H^{4}((0,1), \mathbb{R})$ that satisfy (6) and (7). Then for every $\psi_{0}, \psi_{f} \in H_{(V)}^{4}((0,1), \mathbb{C}) \cap \mathcal{S}$, there exists $T>0$ and $u \in L^{2}((0, T), \mathbb{R})$ such that the solution of (1) satisfies $\psi(T)=\psi_{f}$.

Proof of Corollary 1: Starting from an initial condition $\psi_{0} \in H_{(0)}^{3}$, we first use a control $u \in L^{2}\left(\left(0, T_{1}\right), \mathbb{R}\right)$ to reach a function $\psi\left(T_{1}\right) \in H_{(V)}^{4}(0,1)$, thanks to the second statement of Theorem 1 . Then, by the previous proposition, there exists a control $u \in L^{2}\left(\left(T_{1}, T_{2}\right), \mathbb{R}\right)$ that steers the solution from $\psi\left(T_{1}\right)$ to $\psi\left(T_{2}\right)=\varphi_{1, V}$.

Given a target $\psi_{f} \in H_{(0)}^{3}$, thanks to the previous result and the timereversibility of the Schrodinger equation (i.e. $(\psi, u)$ is a trajectory $\Rightarrow(\bar{\psi}(T-$ $t), u(T-t))$ is a trajectory) there exists $u \in L^{2}\left(\left(T_{2}, T_{3}\right), \mathbb{R}\right)$ that steers the solution from $\psi\left(T_{2}\right)=\varphi_{1, V}$ to $\psi\left(T_{3}\right)=\psi_{f}$.

\section{References}

[1] J.M. Ball, J.E. Marsden, and M. Slemrod. Controllability for distributed bilinear systems. SIAM J. Control Optim., 20, 1982.

[2] K. Beauchard. Local Controllability of a 1-D Schrödinger equation. J. Math. Pures Appl., 84:851-956, July 2005.

[3] K. Beauchard and C. Laurent. Local controllability of 1D linear and nonlinear Schrödinger equations. J. Math. Pures Appl., 94(5):520-554, 2010.

[4] U. Boscain, M. Caponigro, T. Chambrion, and M. Sigalotti. A weak spectral condition for the controllability of the bilinear Schrödinger equation with application to the control of a rotating planar molecule. Comm. Math. Phys., 311(2):423-455, 2012. 
[5] U. Boscain, T. Chambrion, and M. Sigalotti. On some open questions in bilinear quantum control. Proceeding ECC, 2013.

[6] U. Boscain, M.Caponigro, and M. Sigalotti. Multi-input Schrödinger equation: controllability, tracking and application to the quantum angular momentum. Journal of Differential Equations, 256(11):3524-3551, 2014.

[7] N. Boussaid, M. Caponigro, and T. Chambrion. Regular propagators of bilinear quantum systems. preprint (hal-01016299), 2014.

[8] T. Chambrion, P. Mason, M. Sigalotti, and U. Boscain. Controllability of the discrete-spectrum Schrödinger equation driven by an external field. Ann. Inst. H. Poincaré Anal. Non Linéaire, 26(1):329-349, 2009.

[9] Global exact controllability in infinite time of Schrödinger equation: multidimensional case. V. Nersesyan and H. Nersisyan. J. Math. Pures Appl., 97(4):295-317, 2012.

[10] A. Haraux. Séries lacunaires et contrôle semi-interne des vibrations d'une plaque rectangulaire. J. Math. Pures et Appl., 68:457-465, 1989.

[11] Morancey M. Simultaneous local exact controllability of 1D bilinear Schrödinger equations Morgan Morancey . Annales de l'Institut Henri Poincare (C) Non Linear Analysis, 31(3):501-529, May-June 2014.

[12] Morancey M. and Nersesyan V. Global exact controllability of 1d Schrödinger equations with a polarizability term. Comptes Rendus Mathematique, 352(5):425-429, May 2014.

[13] Morancey M. and Nersesyan V. Simultaneous global exact controllability of an arbitrary number of 1d bilinear Schrödinger equations. Journal de Mathématiques Pures et Appliquées, 103(1):228-254, 2015.

[14] V. Nersesyan. Growth of Sobolev norms and controllability of the Schrödinger equation. Comm. Math. Phys., 290(1):371-387, 2009.

[15] V. Nersesyan. Global approximate controllability for Schrödinger equation in higher Sobolev norms and applications. Ann. I. H. Poincaré-AN, 27:901$915,2010$.

[16] J. Pöschel and E. Trubowitz. Inverse Spectral Theory, Pure Appl. Math., volume 130. Academic Press Inc., Boston, MA, 1987.

[17] Jean-Pierre Puel. A regularity property for Schrödinger equations on bounded domains. Rev. Mat. Complut., 26(1):183-192, 2013.

[18] G. Turinici. On the controllability of bilinear quantum systems. In C. Le Bris and M. Defranceschi, editors, Mathematical Models and Methods for Ab Initio Quantum Chemistry, volume 74 of Lecture Notes in Chemistry, Springer, 2000. 